\title{
Mutual projectile-target ionization via the two-center dielectronic interaction in relativistic ion-atom collisions
}

\author{
A.B.Voitkiv, B.Najjari and J.Ullrich \\ Max-Planck-Institut für Kernphysik, Saupfercheckweg 1, D-69117 Heidelberg, Germany
}

\begin{abstract}
We study mutual ionization in relativistic collisions between hydrogen-like projectiles and helium atoms: $\mathrm{X}^{Z+}(1 \mathrm{~s})+\mathrm{He}\left(1 \mathrm{~s}^{2}\right)$ $\rightarrow \mathrm{X}^{(Z+1)+}+\mathrm{He}^{+}(1 s)+2 \mathrm{e}^{-}$. At high collision velocities and for not too heavy projectiles, $2 Z / v \ll 1(v$ is the collision velocity), the mutual ionization proceeds via the direct interaction between two electrons bound (initially) to different colliding particles. Considering for the first time this fundamental process in the case of relativistic collisions, we calculate ionization cross sections and discuss manifestations of relativistic effects. In particular, we predict two novel and interesting phenomena: i) considerable relativistic effects in collisions with low Lorentz factors $\gamma$ and ii) the rapid saturation of these effects at higher $\gamma$. Estimates show that the predicted effects can be experimentally tested using existing facilities and spectrometers.
\end{abstract}

PACS numbers: $34.10+\mathrm{x}, 34.50 . \mathrm{Fa}$

The role of the electron-electron interaction in nature can hardly be overestimated. Although this interaction may be of crucial importance for a vast amount of various phenomena studied by different branches of science ranging from astronomy to biology, some of the perhaps most simple and clear manifestations of this interaction can be observed in ion-atom collisions.

If a bare projectile-nucleus collides with a neutral target-atom, which is initially in the ground state, the atom can be excited/ionized by the interaction with the projectile or/and one or more atomic electrons can be picked up by the nucleus and form bound or lowlying continuum states of the corresponding projectileion. Within such complex reactions the electron-electron interaction can, for instance, be responsible for multipleelectron ionization of atoms in very fast collisions with low charged projectiles. Note, however, that this interaction per se cannot trigger the above reactions.

If initially the projectile is not a fully stripped ion but carries one or more electrons, then in collisions with atomic targets not only atomic electrons but also those of the projectile-ion can be excited and/or lost. During the collision the motion of the atomic electrons can be coupled to the motion of the ionic electrons via the interaction between electrons belonging to different colliding centers and the latter can manifest itself in different ways. In particular, this interaction can directly lead to excitation and/or ionization. Moreover, already just the single interaction between two electrons belonging to the different colliding centers, the so called two-center dielectronic interaction [1] (TCDI), may result in mutual ionization of the projectile and the target that can be viewed as an excellent example of 'scoring two hits with one bullet'.

In nonrelativistic collisions the dynamics of mutual projectile-target ionization via the TCDI has been extensively explored experimentally and theoretically on the levels of both total and differential cross sections (see for reviews [1]-[3], for very recent advances in this field see [4]). The situation, however, is very different for the relativistic domain of collision energies [5]. In- deed, concerning the theory one can note that even the basic formulations of relativistic approaches for treating collisions between an ion and an atom both carrying active electrons have been established only very recently [6]. Besides, we are not aware about any experimental studies of the TCDI in relativistic collisions.

In this Letter, for the first time, we explore theoretically the TCDI in the case of mutual projectile-target ionization at relativistic collision velocities. From the theoretical point of view the simplest and most basic situation for studying this interaction would be to consider collisions of hydrogen-like ions with hydrogen atoms where the internal states of both colliding particles are exactly known. However, in order to deal with such a situation where theoretical predictions can be much easier verified experimentally, we restrict here our consideration to relativistic collisions between hydrogen-like projectiles (with relatively low atomic numbers) and a helium target where, as the result of the collision, one electron is emitted from each of the colliding centers: $\mathrm{X}^{Z+}(1 \mathrm{~s})+$ $\mathrm{He}\left(1 \mathrm{~s}^{2}\right) \rightarrow \mathrm{X}^{(Z+1)+}+\mathrm{He}^{+}(1 s)+2 \mathrm{e}^{-}$.

We assume that in the target frame the incident projectile has a velocity $\mathbf{v}=(0,0, v)$ which can be comparable to the speed of light $c \simeq 137$ a.u. and, hence, the Lorentz factor $\gamma=1 / \sqrt{1-v^{2} / c^{2}}$ may noticeably exceed unity. At high collision velocities and for not too heavy projectiles, where $2 Z / v \ll 1$, the mutual ionization proceeds via the TCDI whereas the second order process, in which the electron of the ion and that of the atom are kicked out by two independent interactions with the nuclei of the atom and ion, respectively, is of minor importance. Thus, the process under consideration represents a very clear example of ionization where the TCDI is not masked and therefore one has favourable conditions for studying relativistic peculiarities in this interaction.

The general form of the transition $S$-matrix element, which describes collisions of atomic particles interacting via the electromagnetic field, is given by (see e.g. [7])

$$
S_{f i}=-\frac{i}{c} \int d^{4} x \int d^{4} y J_{\mu}^{I}(x) D^{\mu \nu}(x-y) J_{\nu}^{A}(y) .
$$


Here, $J_{\mu}^{I}(x)$ and $J_{\nu}^{A}(y)(\mu, \nu=0,1,2,3)$ are the electromagnetic transition 4-currents generated by the projectile-ion at a space-time point $x$ and by the targetatom at a space-time point $y$, respectively, and $D^{\mu \nu}(x-y)$ is the propagator of the electromagnetic field which transmits the projectile-target interaction. In (1) the summation over repeated greek indices is implied. Using Eq.(1) one can show that, within the first order approximation in the projectile-target interaction, the fully differential cross section for the mutual ionization of the target and projectile in a single collision event is given by

$$
\begin{array}{r}
\frac{d \sigma}{d^{3} \mathbf{k}_{a} d^{3} \mathbf{k}_{i} d^{2} \mathbf{Q}_{t r}}=\frac{4}{v^{2}} \frac{1}{\left(\mathbf{q}_{a}^{2}-\left(\varepsilon_{k_{a}}-\varepsilon_{0}\right)^{2} / c^{2}\right)^{2}} \times \\
\left|\left(F_{0}^{I}-\frac{v}{c} F_{3}^{I}\right)\left(F_{A}^{0}-\frac{v}{c} F_{A}^{3}\right)+\frac{F_{3}^{I} F_{A}^{3}}{\gamma^{2}}+\frac{F_{1}^{I} F_{A}^{1}+F_{2}^{I} F_{A}^{2}}{\gamma}\right|^{2}
\end{array}
$$

Here, $\varepsilon_{0}$ and $\varepsilon_{k_{a}}$ are initial and final internal energies of the atom, respectively, $\mathbf{k}_{a}$ is the 3 -momentum of the electron emitted from the atom and $\mathbf{q}_{a}$ is the 3 -momentum transferred to the atom; all the quantities are given in the atomic rest frame. We also introduce quantities $\epsilon_{0}, \epsilon_{k_{i}}$, $\mathbf{k}_{i}$ and $\mathbf{q}_{i}$ which have similar meanings but are for the ion and given in the ion frame [8]. The momentum transfers are defined by $\mathbf{q}_{a}=\left(\mathbf{Q}_{t r}, q_{\text {min }}^{a}\right)$ and $\mathbf{q}_{i}=\left(-\mathbf{Q}_{t r},-q_{\text {min }}^{i}\right)$, where $\mathbf{Q}_{t r}$ is the two-dimensional part of the momentum transferred to the atom, which is perpendicular to the collision velocity $\mathbf{v}$, and the components of the momentum transfers along the collision velocity read

$$
\begin{aligned}
q_{\text {min }}^{a} & =\frac{\varepsilon_{k_{a}}-\varepsilon_{0}}{v}+\frac{\epsilon_{k_{i}}-\epsilon_{0}}{v \gamma} \\
q_{\text {min }}^{i} & =\frac{\epsilon_{k_{i}}-\epsilon_{0}}{v}+\frac{\varepsilon_{k_{a}}-\varepsilon_{0}}{v \gamma} .
\end{aligned}
$$

The inelastic 4-component form-factors of the ion (in the ion frame) and of the atom (in the atom frame) are given by

$$
\begin{aligned}
& F_{0}^{I} \equiv F_{0}^{I}\left(\mathbf{k}_{i}, \mathbf{q}_{i}\right)=-\left\langle\psi_{f}\left|\exp \left(i \mathbf{q}_{i} \cdot \mathbf{r}\right)\right| \psi_{i}\right\rangle \\
& F_{l}^{I} \equiv F_{l}^{I}\left(\mathbf{k}_{i}, \mathbf{q}_{i}\right)=\left\langle\psi_{f}\left|\exp \left(i \mathbf{q}_{i} \cdot \mathbf{r}\right) \alpha_{l}\right| \psi_{i}\right\rangle
\end{aligned}
$$

and

$$
\begin{aligned}
& F_{A}^{0} \equiv F_{A}^{0}\left(\mathbf{k}_{a}, \mathbf{q}_{a}\right)=-\left\langle u_{f}\left|\sum_{j=1}^{2} \exp \left(i \mathbf{q}_{a} \cdot \boldsymbol{\xi}_{j}\right)\right| u_{i}\right\rangle \\
& \left.F_{A}^{l} \equiv F_{A}^{l}\left(\mathbf{k}_{a}, \mathbf{q}_{a}\right)=-\left\langle u_{f}\left|\sum_{j=1}^{2} \alpha_{l(j)} \exp \left(i \mathbf{q}_{a} \cdot \boldsymbol{\xi}_{j}\right)\right| u_{i}\right\rangle, 5\right)
\end{aligned}
$$

respectively $(l=1,2,3)$. In Eq.(4) $\psi_{i}=\psi_{i}(\mathbf{r})$ and $\psi_{f}=\psi_{f}(\mathbf{r})$ are the initial and final internal states of the ion, $\mathbf{r}$ the coordinates of the ion electron with respect to the ion nucleus and $\alpha_{l}$ the Dirac matrices for the electron of the ion. In Eq.(5)) $u_{i}=u_{i}\left(\boldsymbol{\xi}_{1}, \boldsymbol{\xi}_{2}\right)$ and $u_{f}=u_{f}\left(\boldsymbol{\xi}_{1}, \boldsymbol{\xi}_{2}\right)$ are the initial and final internal states of the atom, $\boldsymbol{\xi}_{j}$ are the coordinates of the $j$-th atomic electron with respect to the atomic nucleus and $\alpha_{l(j)}$ are the
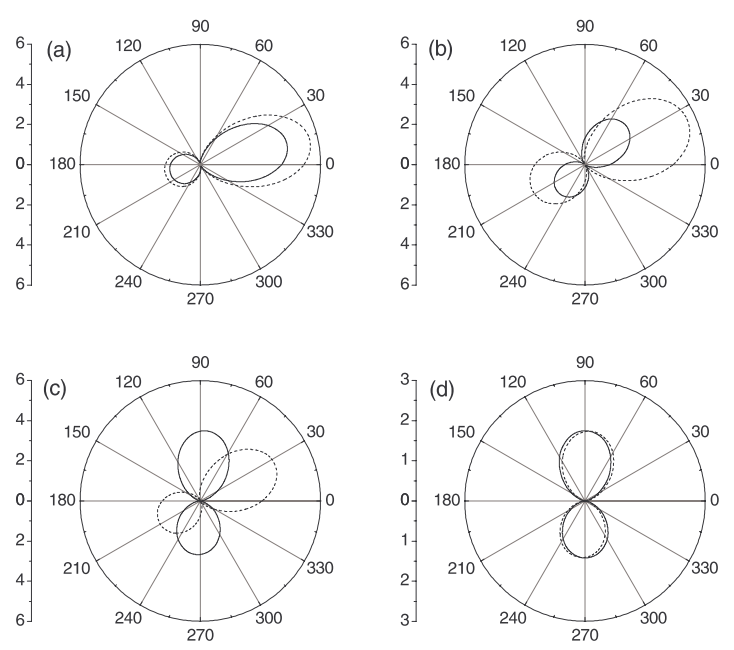

FIG. 1: (a)-(c): 'Fully' differential cross section (in arb. units), as a function of the polar emission angle $\vartheta_{a}=\arccos \left(\mathbf{k}_{a} \cdot \mathbf{v} /\left(k_{a} v\right)\right.$, in the coplanar geometry for $\mathrm{C}^{5+}(1 \mathrm{~s})+\mathrm{He}\left(1 \mathrm{~s}^{2}\right) \rightarrow \mathrm{C}^{6+}+\mathrm{He}^{+}(1 \mathrm{~s})+$ $2 \mathrm{e}^{-}$collisions. $\varepsilon_{k_{a}}=5 \mathrm{eV}, \mathbf{Q}_{t r}$ is pointing to $90^{\circ}$ and its absolute value is 0.1 a.u.. (a) $100 \mathrm{MeV} / \mathrm{u}$ ( $v \simeq 60$ a.u., $\gamma \simeq 1.11)$; (b) 1 $\mathrm{GeV} / \mathrm{u}(v \simeq 120$ a.u., $\gamma \simeq 2.07)$; (c) $23 \mathrm{GeV} / \mathrm{u}(\gamma \simeq 26)$. (d): Fully differential cross section (in arb. units) in the coplanar geometry for single ionization of helium by a free electron impact, $\varepsilon_{k_{a}}=5$ $\mathrm{eV}, Q_{t r}=0.1 \mathrm{a} . \mathrm{u}$. and $\gamma=10^{3}$. Results of relativistic $(c \simeq 137)$ and nonrelativistic $(c=\infty)$ calculations are depicted by solid and dashed curves, respectively.

Dirac matrices for the $j$-th atomic electron. In our calculations initial and final states of helium are described in a Hartree-Fock approximation. Such a treatment has been chosen because it was quite successful in the descriptions of single ionization of helium by fast point-like charged particles [9].

The cross section (2) contains the most detailed information about the TCDI and, besides, is most simple for calculations. One should take into account, however, that even though 'state-to-the-art' experimental techniques allow one to resolve rather well final states of the target fragments it is rather difficult to analyze the fragments of a very fast projectile. At the same time the charge state of the residual projectile-ion can be easily detected in experiment. Therefore, leaving a more detailed exploration of the TCDI in relativistic collisions to a forthcoming article, here we focus our attention on cross sections, which will be measurable in experiments at the GSI (Darmstadt, Germany) scheduled to start in 2004 , and begin our discussion with considering the 'fully' differential cross section for target ionization, $\frac{d \sigma}{d^{3} \mathbf{k}_{a} d^{2} \mathbf{Q}_{t r}}$, where the integration is performed over all possible final continuum states of the electron emitted from the projectile. This cross section is displayed in Figs. 1a-c for $\mathrm{C}^{5+}(1 \mathrm{~s})+\mathrm{He}\left(1 \mathrm{~s}^{2}\right) \rightarrow \mathrm{C}^{6+}+\mathrm{e}^{-}+\mathrm{He}^{+}(1 \mathrm{~s})+\mathrm{e}^{-}$ collisions in the case of coplanar geometry in which the momentum of the emitted electron lies in the plane defined by the vectors of the incident projectile velocity and the momentum transfer and where the 'fully' differential 
cross section reaches its largest values. The energy of the electron emitted from $\mathrm{He}$ is taken to be $5 \mathrm{eV}$ and the transverse momentum transfer is $Q_{t r}=0.1$ a.u.. Figs. 1a-c show that relativistic effects in single ionization of helium via the TCDI can be quite noticeable already at $\gamma \simeq 1.1$ and may become very substantial at $\gamma \simeq 2$ and higher. It is interesting to note that such pronounced manifestations of the relativistic effects in the target ionization by a 'bound' electron are in sharp contrast to the case of ionization by a free electron with the same $Q_{t r}=0.1$ a.u.. It is seen in Fig. 1d that in the latter case even at $\gamma=10^{3}$ the fully differential cross section is still very weakly influenced by the relativistic effects [10].

The relativistic effects in the TCDI can be split into those depending on the collision velocity $v$ and disappearing when $v / c \ll 1$ and those which are connected with relativistic effects in the inner motions of electrons within each of the colliding centers. The latter effects are reflected by the coupling between the space components $(l=1,2,3)$ of the corresponding form-factors in (2). Although formally they do not disappear even when $v / c \ll 1$, their influence on collisions between light atomic systems is quite weak. Relativistic effects, which are related to the collision velocity, are much more important for the collision system under consideration. They include the following: i) the retardation effect which in the target frame is reflected by the term $\left(\varepsilon_{k_{a}}-\varepsilon_{0}\right)^{2} / c^{2}$ in the denominator of Eq.(2); ii) the longitudinal components $q_{\min }^{a}$ and $q_{\text {min }}^{i}$, due to the presence of the Lorentz factor, become unequal and depend differently on the transition energies $\varepsilon_{k_{a}}-\varepsilon_{0}$ and $\epsilon_{k_{i}}-\epsilon_{0}$; iii) the coupling between the zeroth and third components of the formfactors in Eq.(2). Note that for the rather asymmetric collision system considered in Figs. 1a-c the above point ii) manifests itself very clearly.

Valuable information about the collision process can also be obtained by considering cross section differential in energy and solid angle of the emitted electron, $\frac{d^{2} \sigma}{2 \pi d \varepsilon_{k_{a}} \sin \vartheta_{a} d \vartheta_{a}}$. This cross section for $\mathrm{O}^{7+}(1 \mathrm{~s})+\mathrm{He}\left(1 \mathrm{~s}^{2}\right)$ $\rightarrow \mathrm{O}^{8+}+\mathrm{He}^{+}(1 \mathrm{~s})+2 \mathrm{e}^{-}$collisions is depicted in Figs. $2 \mathrm{a}-\mathrm{d}$ where it is given as a function of the polar emission angle $\vartheta_{a}$ of the electron for a fixed emission energy of $5 \mathrm{eV}$. At relatively low values of $\gamma$ relativistic effects increase with increasing collision energy. However, starting with $\gamma \simeq 6-8$ we observe the 'saturation' of these effects: the shape and absolute values of the spectrum do not change substantially with a further increase in $\gamma$ (compare Figs. 2c and 2d).

In a sharp contrast, such a 'saturation' would not hold for spectra of electrons emitted from targets by a free electron impact. In the latter case even for ultrarelativistic collisions both the magnitude and the shape of the spectra depend on $\gamma$. In collisions with a free electron the $\gamma$-dependence arises due to the contribution of very small momentum transfers (or very large impact parameters) and has its origin in the Lorentz contraction of the electromagnetic field generated by relativistic pointlike charges: compared to nonrelativistic predictions the

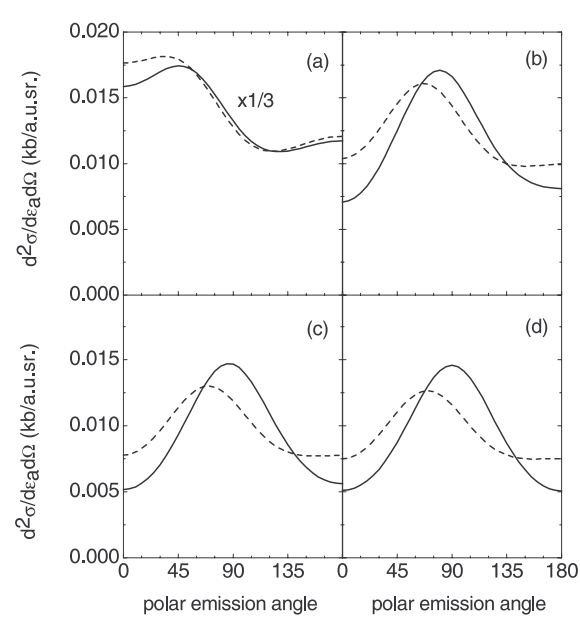

FIG. 2: Angular distribution of $5 \mathrm{eV}$ electrons emitted from helium in $\mathrm{O}^{7+}(1 \mathrm{~s})+\mathrm{He}\left(1 \mathrm{~s}^{2}\right) \rightarrow \mathrm{O}^{8+}+\mathrm{He}^{+}(1 \mathrm{~s})+2 \mathrm{e}^{-}$collisions. (a) $100 \mathrm{MeV} / \mathrm{u}$; (b) $1 \mathrm{GeV} / \mathrm{u}$; (c) $5.6 \mathrm{GeV} / \mathrm{u}(\gamma \simeq 6)$; (d) $1 \mathrm{TeV} / \mathrm{u}$ $(\gamma \approx 1000)$. Results of relativistic and nonrelativistic calculations are depicted by solid and dashed curves, respectively.

range of this field is effectively increased by a factor of $\gamma$ in the transverse direction.

The basic physical reason, why large impact parameters (or small $Q_{t r}$ ) are essentially cut off in the target ionization via the TCDI, is that the internal state of the projectile is also excited in the collision. In particular, this excitation manifests itself in the following two important points. Firstly, as it can be shown by using the conservation of the projectile transition 4-current, at $\gamma \gg\left(\varepsilon_{k_{a}}-\varepsilon_{0}\right) /\left(\epsilon_{k_{i}}-\epsilon_{0}\right)$ one has $F_{0}^{I}-\frac{v}{c} F_{3}^{I}=-\left\langle\psi_{f}\left|\exp \left(i \mathbf{q}_{i} \cdot \mathbf{r}\right)\right| \psi_{i}\right\rangle\left(\frac{1}{\gamma^{2}}+\frac{v^{2}\left(\varepsilon_{k_{a}}-\varepsilon_{0}\right)}{c^{2} \gamma\left(\epsilon_{k_{i}}-\epsilon_{0}\right)}\right)+$ $\frac{v^{2} / c}{\epsilon_{k_{i}}-\epsilon_{0}}\left\langle\psi_{f}\left|\exp \left(i \mathbf{q}_{i} \cdot \mathbf{r}\right) \mathbf{Q}_{t r} \cdot \boldsymbol{\alpha}_{t r}\right| \psi_{i}\right\rangle$, where $\boldsymbol{\alpha}_{t r}=\left(\alpha_{1}, \alpha_{2}\right)$. Thus, the first addendum in the second line of Eq.(2) is small if $\gamma$ is high and $Q_{t r}$ is low. Besides, due to the factors $\gamma^{-1}$ and $\gamma^{-2}$, the last two addenda in that line are also small at high $\gamma$. Note that Eq.(2) can be used also for the treatment of target ionization by a point-like charged projectile with a charge $Z_{p}$ if we replace $F_{0}^{I}$ by $Z_{p}$ and set $F_{1}^{I}=F_{2}^{I}=F_{3}^{I}=0$ and $\epsilon_{k_{i}}-\epsilon_{0}=0$. Considering the corresponding couplings of the target and projectile form-factors in Eq.(2) we see that, compared to the ionization by a point-like projectile, at high enough $\gamma$ the target ionization via the TCDI in collisions with small $Q_{t r}$ is strongly suppressed. Secondly, let us compare the photon propagator for target ionization by a point-like charged projectile, given by $G_{1} \sim\left(Q_{t r}^{2}+\left(\varepsilon_{k_{a}}-\varepsilon_{0}\right)^{2} /\left(v^{2} \gamma^{2}\right)\right)^{-1}$, with the photon propagator in Eq.(2), $G_{2} \sim\left(\mathbf{q}_{a}^{2}-\left(\varepsilon_{k_{a}}-\varepsilon_{0}\right)^{2} / c^{2}\right)^{-1} \equiv$ $\left(Q_{t r}^{2}+\frac{\left(\varepsilon_{k_{a}}-\varepsilon_{0}+\epsilon_{k_{i}}-\epsilon_{0}\right)^{2}}{v^{2} \gamma^{2}}+2(\gamma-1) \frac{\left(\varepsilon_{k_{a}}-\varepsilon_{0}\right)\left(\epsilon_{k_{i}}-\epsilon_{0}\right)}{v^{2} \gamma^{2}}\right)^{-1}$.

At fixed $Q_{t r}$ one has $G_{2}>G_{1}$ because the length of the 4-momentum of the photon corresponding to $G_{2}$ deviates stronger from the mass-shell condition 


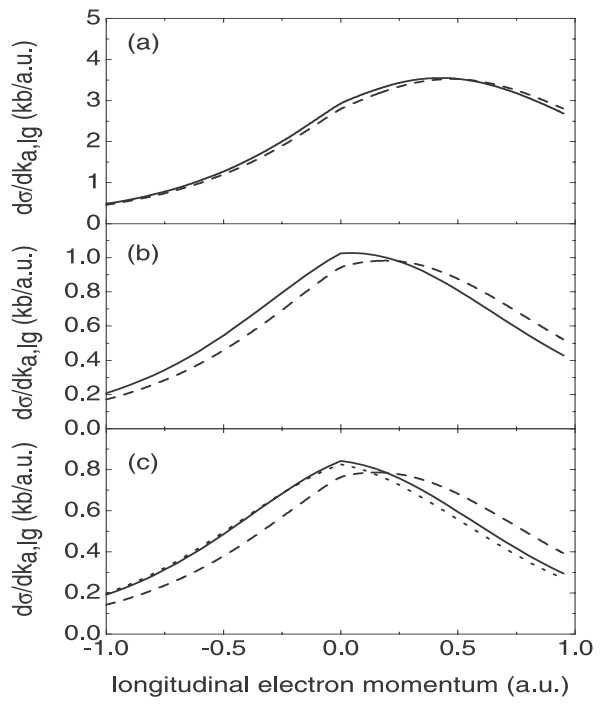

FIG. 3: Longitudinal momentum distribution of electrons emitted from helium in $\mathrm{C}^{5+}(1 \mathrm{~s})+\mathrm{He}\left(1 \mathrm{~s}^{2}\right) \rightarrow \mathrm{C}^{6+}+\mathrm{He}^{+}(1 \mathrm{~s})+2 \mathrm{e}^{-}$ collisions. (a) $100 \mathrm{MeV} / \mathrm{u}$; (b) $1 \mathrm{GeV} / \mathrm{u}$; (c) $5.6 \mathrm{GeV} / \mathrm{u}$. Results of relativistic and nonrelativistic calculations are depicted by solid and dashed curves, respectively. In addition, the dotted curve in (c) shows results of relativistic calculations for $1 \mathrm{TeV} / \mathrm{u}$.

$q^{2}=0$. Such a more pronounced virtuality of the photon transmitting the TCDI also reduces the range of this interaction compared to the range of the interaction with a point-like charged projectile.

Amongst singly differential cross sections, the one that is differential in the longitudinal component $k_{a, l g}=$ $\mathbf{k}_{a} \cdot \mathbf{v} / v$ of the target electron momentum, $\frac{d \sigma}{d k_{a, l g}}$, often provides most important information about the collision dynamics. This cross section is displayed in Figs. 3a-c for the same collision system as considered in Figs. 1ac. Nonrelativistic calculations suggest a strong forwardbackward asymmetry in the longitudinal electron spectrum even at $v \rightarrow c$. However, relativistic calculations predict that this asymmetry should be less pronounced even at moderate values of $\gamma$ and that it can be substantially reduced when $\gamma$ increases. Similarly to the consideration of the doubly differential cross section, we observe that for the collision system in question the relativistic effects in the longitudinal emission spectrum saturate at about $\gamma \sim 5-7$ and that a further increase in $\gamma$ influences quite weakly this spectrum.

In conclusion, we have explored the two-center dielectronic interaction in mutually ionizing collisions between not too heavy hydrogen-like relativistic projectile-ions and helium atoms. We have calculated different cross sections and found interesting effects arising due to collision velocities approaching the speed of light. In particular, two novel phenomena have been predicted for the target ionization by a 'bound' electron: considerable relativistic effects in collisions with low $\gamma$-s and their 'saturation' already at not very high $\gamma$-s. Both these phenomena would not hold for helium ionization by a free electron and are quite unexpected from the point of view of the physics of ionization of light targets by relativistic point-like charged projectiles. For collision energies not exceeding $\sim 1 \mathrm{GeV} / \mathrm{u}$ our predictions will be experimentally tested in the near future. The results for higher collision energies, including the conclusion about the existence of the 'saturation' region, could be verified experimentally after upgrading the accelerator facilities at the GSI where one will be able to accelerate ions to collision energies corresponding to $\gamma \lesssim 30$.
[1] N. Stolterfoht, R.D. DuBois and R.D. Rivarola, Electron Emission in Heavy Ion-Atom Collisions (Springer, 1997)

[2] E.C.Montenegro, W.E.Meyerhof and J.H.McGuire, Adv.At.Mol. and Opt. Phys. 34249 (1994)

[3] J.H. McGuire Electron Correlation Dynamics in Atomic Collisions (Cambridge University Press, 1997)

[4] H. Kollmus, R. Moshammer, R.E. Olson, S. Hagmann, M. Schultz and J. Ullrich, Phys. Rev. Lett. 88103202 (2002)

[5] J.Eichler and W.E.Meyerhof Relativistic Atomic Collisions (Academic Press, 1995)

[6] A.B.Voitkiv, N.Grün and W.Scheid, Phys.Rev. A 61 052704 (2000); A.B.Voitkiv, M.Gail and N.Grün, J.Phys. B 331299 (2000); A.B. Voitkiv, Phys. Rep. 392191 (2004)
[7] J.D.Bjorken and S.D.Drell Relativistic Quantum Mechanics (McGraw-Hill, 1964)

[8] We note that the denominator in Eq.(2) can be rewritten in an equivalent form by taking into account that $\mathbf{q}_{a}^{2}-$ $\left(\varepsilon_{k_{a}}-\varepsilon_{0}\right)^{2} / c^{2} \equiv \mathbf{q}_{i}^{2}-\left(\epsilon_{k_{i}}-\epsilon_{0}\right)^{2} / c^{2}$.

[9] D. Madison, Phys. Rev. A 82449 (1973); L. Gulyas, P.D. Fainstein, and A. Salin, J. Phys. B 28245 (1995); D. Madison et al, J. Phys. B 353297 (2002)

[10] Note that at such a relatively large momentum transfer like $Q_{t r}=0.1$ a.u. the relativistic effects in the fully differential cross section for single ionization of helium by a free electron impact practically do not increase even in the limit $\gamma \rightarrow \infty$. 